\title{
Usabilidade de um dashboard destinado à autorregulação de estudantes em Sala de Aula Invertida
}

\author{
João Carlos Sedraz Silva - UNIVASF - joao.sedraz@univasf.edu.br \\ Rodrigo Lins Rodrigues - UFRPE - rodrigo.linsrodrigues@ufrpe.br \\ Jorge Luis Cavalcanti Ramos - UNIVASF - jorge.cavalcanti@ univasf.edu.br \\ Erik de Gouveia Zambom - UFPE - egz@cin.ufpe.br \\ Fernando da Fonseca de Souza - UFPE - fdfd@cin.ufpe.br
}

\begin{abstract}
Resumo. O presente trabalho teve por objetivo avaliar a usabilidade de um dashboard destinado à Autorregulação da Aprendizagem de estudantes em Sala de Aula Invertida. O método adotado contemplou a convergência de múltiplas fontes de evidência, com a triangulação de dados coletados por meio do questionário SUS e de observação. Segundo a opinião de trinta e nove usuários que colaboraram com a pesquisa, mesmo com algumas sugestões de melhoria, a usabilidade do recurso avaliado é satisfatória. Além disso, os resultados apontam que o artefato pode ser utilizado para a promoção da Autorregulação da Aprendizagem na Sala de Aula Invertida, principalmente, na autorreflexão do estudante sobre o seu comportamento no contexto educacional.
\end{abstract}

Palavras-chave: Tecnologia Educacional, Feedback, Sala de Aula Invertida.

Abstract. This study aimed to evaluate the usability of a dashboard dedicated to SelfRegulation Learning of students in Flipped Classroom. The adopted method contemplated the convergence of multiple sources of evidence, with the triangulation of data collected through the SUS questionnaire and observation. According to the opinion of thirty-nine users who collaborated with the research, even with some suggestions for improvement, the usability of the evaluated resource is satisfactory. Addition, the artifact can be used to promote Self-Regulation Learning in the Flipped Classroom, mainly, on the student's selfreflection about his behavior in the educational context.

Keywords: Educational Technology, Feedback, Flipped Classroom.

\section{Introdução}

Uma das definições mais frequente da metodologia Sala de Aula Invertida (SAI) é "inversão de eventos que, tradicionalmente, ocorriam dentro da sala de aula e passaram a acontecer fora da sala de aula e vice-versa" (Lage et al., 2000). Apesar de justificar o uso da palavra "invertida", essa definição é simples e não apresentam características importantes dessa metodologia. Em trabalhos recentes, a SAI passou a ser definida por outras perspectivas, as quais observam as atribuições dos professores e estudantes, bem como os propósitos de atividades presenciais e a distância.

Para Valente (2014), nessa metodologia a atribuição principal do professor é mediar o processo de aprendizagem, em vez de se concentrar na apresentação de conteúdo, enquanto o estudante é responsável por sua preparação prévia e por seu envolvimento durante as aulas. Segundo Fidalgo-Blanco et al. (2017) e Mazur (2013), a SAI é um modelo específico de Blended Learning (Tori, 2009), o qual combina atividades a distância, com o propósito de transmissão do conhecimento antes das sessões presenciais, e práticas de aprendizagem ativa em sala de aula, voltadas para a assimilação do conteúdo. Essa combinação oferece vários benefícios para os discentes e os docentes.

As atividades a distância tornam o aprendizado mais flexível, permitindo que o estudante acesse o conteúdo no seu próprio ritmo. Por meio dessa flexibilidade, indiretamente, a inversão do processo de aprendizado estimula a autonomia e a 
responsabilidade do estudante, duas características relevantes para o seu desenvolvimento pessoal e profissional (Mason et al., 2013). Um outro benefício desse tipo de atividade, especialmente quando realizada por meio de ambientes virtuais, é fornecer ao professor informações sobre a preparação dos estudantes. A partir dessas informações, o docente pode customizar as suas aulas de acordo com as necessidades dos discentes (Valente, 2014).

Para Delozier e Rhodes (2016), a maior vantagem da instrução direta a distância é proporcionar tempo adicional para uma aprendizagem ativa. Esse tipo de aprendizagem corresponde às práticas que buscam engajar o estudante na sala de aula, como debates, resolução de problemas e trabalho em equipe (Prince, 2004). Em relação às aulas expositivas tradicionais, vários trabalhos demonstram evidências consistentes de que a aprendizagem ativa pode melhorar o desempenho e reduzir a evasão dos discentes (Freeman et al., 2014).

Com as diversas possibilidades para a combinação de atividades a distância e práticas ativas, a SAI torna viável a utilização de estratégias pedagógicas adequadas para vários estilos de aprendizagem (Felder et al., 1988), o que favorece ainda mais a aceitação dessa metodologia pelos estudantes (O'Flaherty e Phillips, 2015).

Apesar dos seus benefícios, a adoção da SAI também apresenta alguns desafios. Assegurar que o estudante se prepare, adequadamente, nas atividades a distância é um desafio bem conhecido da SAI (Mazur, 1997; Sun et al., 2016). Se esta preparação for negligenciada, o discente terá dificuldades para acompanhar as sessões presenciais em sala de aula, comprometendo o seu aprendizado (Karaoglan et al., 2017).

Pesquisas revelam que as atividades a distância exigem um corpo discente com um conjunto de habilidades (Rodrigues et al., 2016; Sun et al., 2016; Karaoglan et al., 2017). Isso significa que o estudante deve ser proativo e obter conhecimento de maneira autodirigida. Além disso, é desejado que o discente seja ativo na definição e busca de objetivos de aprendizagem, que use estratégias específicas para resolver problemas, monitorar seus comportamentos e refletir sobre o desempenho (Sun et al., 2016). Em essência, a necessidade de preparação por meio de atividades a distância promove a Autorregulação da Aprendizagem (ARA) (Zimmerman, 2000) como um desafio crítico para o sucesso da SAI.

Embora exista um reconhecimento do papel da ARA, ainda, é incipiente a pesquisa sobre como ajudar os estudantes a desenvolverem esse tipo de habilidade no contexto da aprendizagem invertida. À medida que cresce a adoção da SAI em instituições de ensino, inclusive com estímulo financeiro de órgãos mantenedores (Capes, 2010, 2015), torna-se cada vez mais importante investigar mecanismos para a promoção da autorregulação dos estudantes envolvidos nesse contexto de aprendizagem. As pesquisas sobre Learning Analytics (LA) representam um campo emergente da Tecnologia Educacional que pode colaborar com esse tipo de investigação, por meio de projetos que envolvem a medição, coleta, análise e comunicação de dados educacionais (Siemens e Baker, 2012).

De acordo com Durall e Gros (2014), as pesquisas de LA oferecem soluções como dashboards, enriquecidos de dados coletados em contextos educacionais, com potencial para auxiliar os discentes em processos de ARA. Alguns trabalhos apresentaram evidências empíricas desse potencial (Corrin e Barba, 2015; Tabuenca et al., 2015; Davis et al., 2016). Mas, mesmo com o crescente número de publicações que reforçam a relevância desses temas, na literatura revisada, não foram encontrados trabalhos com a avaliacão desse potencial por indivíduos inseridos em cenários de SAI. Diante disso, o objetivo deste estudo foi avaliar a usabilidade de um dashboard destinado à Autorregulação da Aprendizagem em Sala de Aula Invertida.

Além desta introdução, o trabalho está organizado com mais quatro seções, as quais apresentam o dashboard que foi avaliado, o método utilizado para a análise da usabilidade do artefato, a discussão dos resultados e as considerações finais da pesquisa. 


\section{Um dashboard dedicado ao apoio da ARA em SAI}

Segundo Verbert et al. (2013), na perspectiva do discente, a aplicação de dashboards no processo de LA pode ser representado pelos quatro (4) estágios indicados na Figura 1. O estágio de percepção está relacionado com os dados educacionais, os quais são coletados de fontes como Sistemas de Gestão da Aprendizagem (LMS, do inglês Learning Management System). Esses dados, geralmente, são tratados e apresentados de forma estruturada, com destaque para informações relevantes sobre o comportamento e o desempenho do usuário. No estágio autorreflexão, intuitivamente, o estudante formula perguntas a respeito da utilidade e relevância dos dados. A compreensão surge das respostas elaboradas pelo discente para as perguntas do estágio anterior. O impacto coincide com o propósito das soluções de LA, o qual é estimular a ARA, gerando um novo significado para o aprendizado ou mudança de comportamento do estudante.

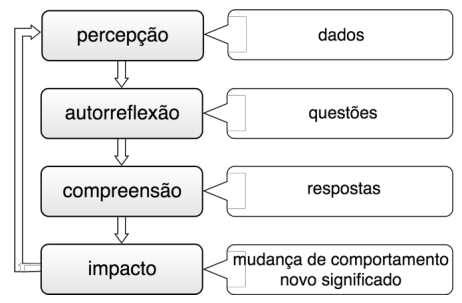

Figura 1. Processo de Learning Analytics (Verbert et al., 2013).

Considerando os estágios sugeridos por Verbert et al. (2013) e trabalhos relacionados a esta pesquisa (Corrin e Barba 2015; Tabuenca et al., 2015; Davis et al., 2016), foi desenvolvido e implementado um dashboard no Amadeus LMS ${ }^{1}$ (Figura 2).

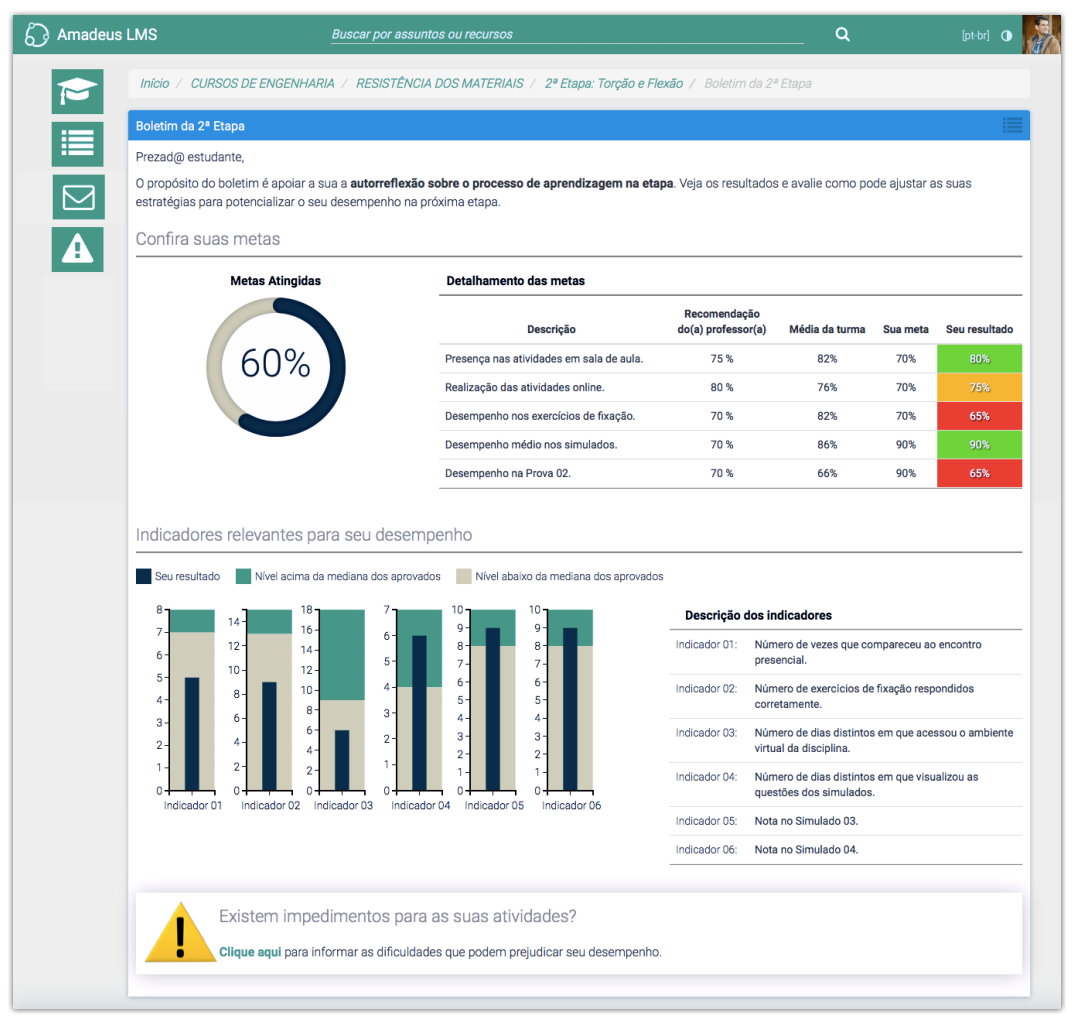

Figura 2. Dashboard avaliado na pesquisa.

${ }^{1}$ https://softwarepublico.gov.br/social/amadeus 
O dashboard avaliado nesta pesquisa funciona como um boletim de uma unidade didática, no qual são estruturadas três seções. Na seção "Confira suas metas", os dados fornecidos pelo discente no início da unidade didática são comparados com os seus resultados e as médias dos seus colegas. Para facilitar a interpretação das informações, são adotados um gráfico de rosca, com o percentual de metas atingidas pelo usuário, e uma tabela que realça os resultados, condicionando a cor das células às metas estabelecidas pelo estudante e aos valores recomendados pelo professor. Ao passar o cursor sobre o resultado, é exibida uma dica de contexto (tooltip), complementando o feedback (Figura 3).

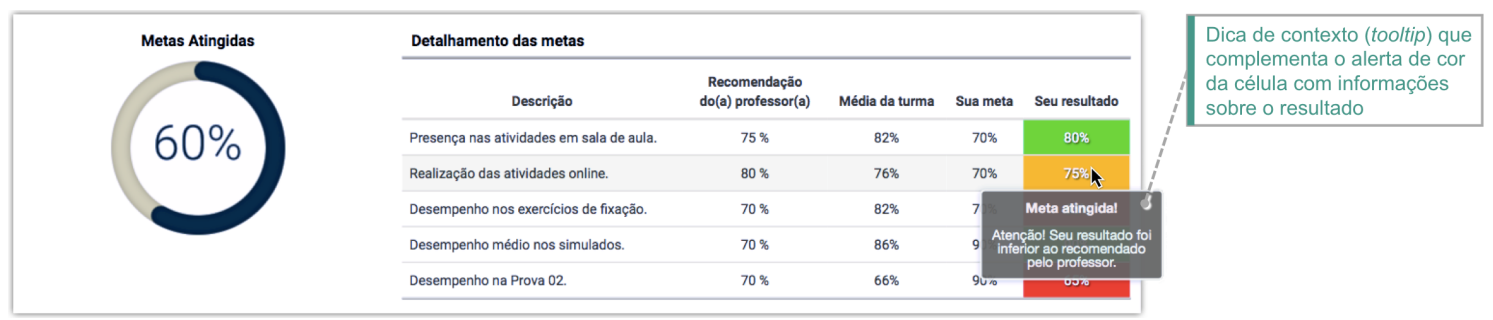

Figura 3 - Detalhamento das metas apresentadas no dashboard.

Na seção "Indicadores relevantes para o seu desempenho", são mostrados gráficos dos indicadores mais correlacionados ao desempenho dos estudantes. Esses indicadores podem ser obtidos por técnicas de seleção de atributos, aplicadas em dados das avaliações e das interações dos participantes da disciplina. Em cada gráfico, são agrupados dados que permitem a comparação social dos discentes, como o valor máximo e a mediana alcançada pelos estudantes aprovados na unidade didática (Figura 4).

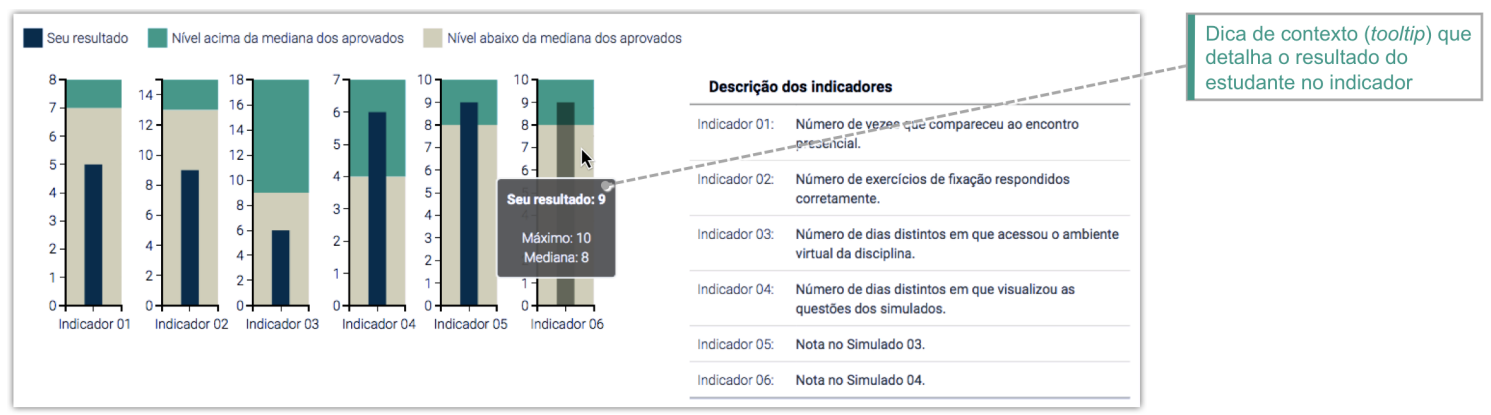

Figura 4 - Gráfico de indicadores do dashboard.

O dashboard, também, oferece um link para que os estudantes possam comunicar as suas dificuldades ao professor. Juntos, esse canal de comunicação, os gráficos e a tabela de detalhamento das metas, podem apoiar o usuário em processos de autorregulatórios (Zimmerman, 2000), especialmente, na autorreflexão do estudante sobre o seu comportamento no contexto educacional.

\section{Método}

Com o objetivo de avaliar o dashboard apontado na seção 2 deste artigo, o artefato foi submetido a testes de usabilidade. Esse tipo de teste são destinados, especialmente, à identificação de problemas nas interfaces e a uma melhor compreensão sobre a interação do usuário com o sistema (Preence et al., 2005). 


\subsection{Participantes}

Os testes foram realizados em setembro de 2017, por trinta e nove (39) estudantes da Universidade Federal do Vale do São Francisco (Tabela 1). No período dos testes, todos os participantes da pesquisa integravam cursos que adotam a metodologia SAI e, assim, representaram usuários potenciais do dashboard implementado no Amadeus LMS.

Tabela 1. Dados do perfil dos estudantes participantes da pesquisa.

\begin{tabular}{l|l|c|c}
\hline \multicolumn{1}{c|}{ Questão } & \multicolumn{1}{c|}{ Alternativa } & $\mathbf{N}^{\star}$ & $\%$ \\
\hline \multirow{2}{*}{ Qual a sua idade? } & Entre 18 e 20 anos & 06 & 15,38 \\
\cline { 2 - 4 } & Entre 21 e 23 anos & 20 & 51,28 \\
\cline { 2 - 4 } & 23 ou mais & 13 & 33,33 \\
\hline \multirow{2}{*}{ Qual o seu sexo? } & Feminino & 11 & 28,21 \\
\cline { 2 - 4 } & Masculino & 28 & 71,79 \\
\hline \multirow{2}{*}{ Qual o seu curso? } & Engenharia Agrícola e Ambiental & 01 & 2,56 \\
\cline { 2 - 4 } & Engenharia de Produção & 06 & 15,38 \\
\cline { 2 - 4 } & Engenharia Elétrica & 32 & 82,05 \\
\hline \multirow{2}{*}{$\begin{array}{l}\text { No seu curso, já participou de disciplinas com a } \\
\text { metodologia Sala de Aula Invertida? }\end{array}$} & Sim & 39 & 100,00 \\
\cline { 2 - 4 } & Não & 00 & 00,00 \\
\hline \multirow{2}{*}{$N=$ Número de estudantes para cada alternativa. } & &
\end{tabular}

\subsection{Procedimentos}

Os testes de usabilidade podem ser conduzidos com o auxílio de diversos procedimentos de coleta e análise de dados, os quais buscam medir o desempenho de usuários típicos em tarefas típicas (Preence et al., 2005). Nesse sentido, os testes realizados contaram com um roteiro específico, relacionado a casos de uso do dashboard (Tabela 2). O roteiro foi organizado para que, partindo da tela inicial do ambiente virtual de uma disciplina, os usuários pudessem concluir os passos planejados em até cinco (5) minutos, proporcionando a coleta de dados essenciais para a avaliação do artefato e evitando um comprometimento excessivo do tempo dos participantes.

Tabela 2. Roteiro de testes.

\begin{tabular}{c|l}
\hline Passo & \multicolumn{1}{c}{ Descrição } \\
\hline 1 & Acesse o recurso "Boletim da Etapa 02". \\
\hline 2 & Quais indicadores o usuário deve melhorar na próxima etapa? \\
\hline 3 & Quais metas não foram atingidas pelo usuário? \\
\hline 4 & Qual a média da turma para o "Desempenho nos exercícios de fixação"? \\
\hline 5 & $\begin{array}{l}\text { No campo "Existem impedimentos para as suas atividades", digite "Muitas disciplinas neste semestre" e } \\
\text { clique no botão "SALVAR" para registrar as informações. }\end{array}$ \\
\hline
\end{tabular}

As sessões de testes aconteceram em um laboratório com computadores, por meio dos quais os participantes da pesquisa tiveram acesso a uma instância do Amadeus LMS alimentada com dados fictícios. Cada sessão seguiu o fluxo indicado na Figura 5 e contou com até sete (7) estudantes, os quais foram orientados e observados por um dos autores desta pesquisa durante, aproximadamente, vinte (20) minutos. Os dados para a avaliação dos artefatos foram coletados por meio de observações e da aplicação do questionário System Usability Scale - SUS (Brooke et al., 1996).

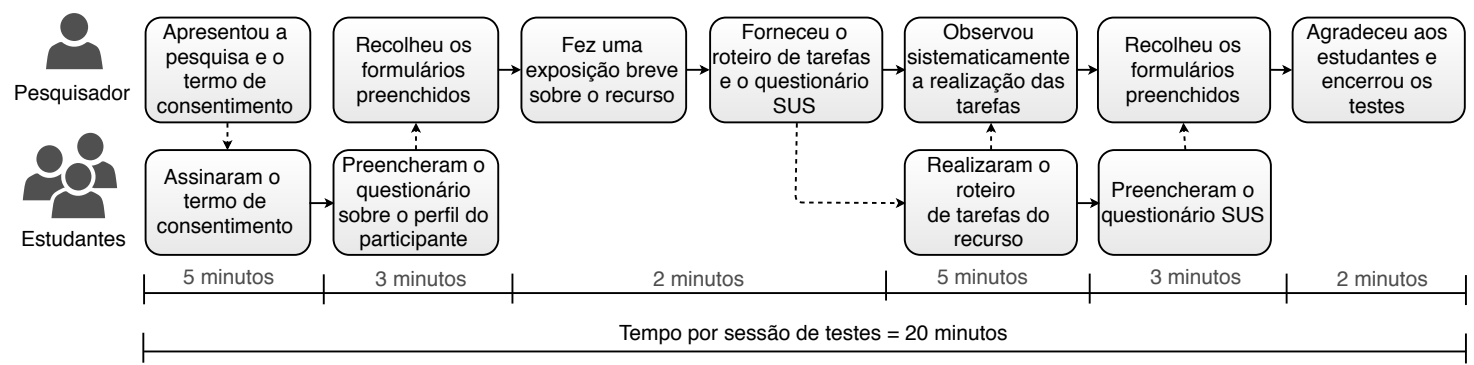

Figura 5. Fluxo da avaliação do recurso implementado no Amadeus LMS.

Nesta pesquisa, as observações foram públicas e sistemáticas (Flick, 2009). No momento em que os estudantes realizaram as tarefas previstas no roteiro, o pesquisador 
registrou os participantes que concluíram os testes dentro do tempo esperado, o número de pedidos de ajuda, os comentários dos estudantes e outras informações relevantes para a avaliação do recurso implementado. Também foram realizadas observações indiretas, a partir do $\log$ do Amadeus LMS e dos resultados das tarefas, registrados pelos estudantes durante a execução do roteiro de teste. As observações indiretas forneceram o tempo de realização das tarefas e o número de erros cometidos por cada participante.

Em relação ao questionário SUS, trata-se de um instrumento calibrado e amplamente utilizado em testes de usabilidade (Assila et al., 2016). Esse questionário é constituído por dez (10) itens. Cada item contém uma declaração relativa a um aspecto de interesse, a qual é acompanhada por uma escala Likert, que varia de 1 - "discordo completamente" até 5 "concordo completamente". As declarações contidas nos itens do SUS contemplam diversos aspectos da usabilidade do sistema avaliado, como satisfação, facilidade de uso, consistência da interface e confiança do usuário.

O SUS produz um resultado representante de uma medida geral da usabilidade do sistema a ser avaliado. Nesse resultado, a contribuição de cada item do questionário está entre 0 e 4. Para os itens ímpares, o valor é calculado subtraindo-se 1 da pontuação atribuída pelo respondente. Para os itens pares, o valor é calculado subtraindo-se de 5 a pontuação atribuída pelo respondente no item. Desse modo, o resultado do SUS é encontrado multiplicando-se a soma das pontuações dos itens por 2,5 (Brooke et al., 1996).

De acordo com Bangor et al. (2009), o resultado do SUS pode ser apresentado de uma forma diferente, por uma classificação referente a intervalos que torna mais fácil a compreensão da avaliação do sistema (Tabela 3). Nesta pesquisa, essa classificação foi adotada para a análise dos dados coletados por meio do SUS.

Tabela 3. Classificação da usabilidade do sistema [Bangor et al. 2009].

\begin{tabular}{c|c|c}
\hline Classificação & Resultado do SUS & Avaliação do sistema \\
\hline A & De 90 a 100 & Excelente \\
\hline B & De 80 a 89 & Bom \\
\hline C & De 70 a 79 & Aceitável \\
\hline D & De 0 a 69 & Com problemas de usabilidade \\
\hline
\end{tabular}

\section{Resultados}

Como descrito na seção anterior, trinta e nove (39) potenciais usuários do dashboard implementado do Amadeus LMS participaram dos testes de usabilidade, nos quais foram coletados dados por meio de observação e da aplicação do questionário SUS. Os resultados da análise desses dados serão discutidos nas próximas seções.

\subsection{Análise dos dados quantitativos da observação}

A partir da observação direta e indireta dos usuários, foram obtidos os dados quantitativos que estão organizados na Tabela 4. Segundo esses dados, todos os participantes conseguiram concluir o roteiro proposto, com um tempo médio de 3 minutos e 7 segundos, sendo que 4 usuários demoraram mais de 5 minutos. Somente 1 usuário cometeu erro, o qual foi causado por uma interpretação equivocada dos gráficos de indicadores.

Tabela 4. Dados quantitativos dos testes $(N=39)$.

\begin{tabular}{l|c}
\hline \multicolumn{1}{c|}{ Descrição da métrica } & Resultado \\
\hline Número de usuários que concluíram o roteiro & $39(100,00 \%)$ \\
\hline Tempo médio para a realização do roteiro (hh/mm/ss) & $00: 03: 07$ \\
\hline Número de usuários que não concluíram o roteiro no tempo esperado (até 5 min) & $4(10,26 \%)$ \\
\hline Número de usuários que cometeram erros & $1(2,56 \%)$ \\
\hline Número de usuários que solicitaram ajuda & $0(0,00 \%)$ \\
\hline
\end{tabular}




\subsection{Análise dos dados quanlitativos da observação}

Durante as sessões de testes, de maneira geral, os usuários fizeram comentários positivos sobre o dashboard, como os transcritos a seguir.

"Eu achei o recurso bastante interessante para o aluno que estuda estabelecendo metas, como também, para que o mesmo tenha o controle sobre os seus resultados."

"Ajuda o estudante a fazer reflexão sobre o desempenho nas disciplinas."

"Recurso bem interativo e de fácil entendimento."

Mas, adicionalmente aos comentários positivos, alguns discentes narraram dificuldades enfrentadas nos testes e, também, apresentaram sugestões que podem favorecer a usabilidade do artefato. Com a codificação desses relatos, notou-se que as dificuldades e sugestões convergiram conforme a Tabela 5.

Tabela 5. Dificuldade e sugestões identificadas.

\begin{tabular}{c|l}
\hline Código & \multicolumn{1}{|c}{ Descrição } \\
\hline D01 & Falta de clareza dos gráficos de indicadores \\
\hline S01 & Ajuste no gráfico de indicadores \\
\hline S02 & Anexos no campo de comunicação dos impedimentos \\
\hline
\end{tabular}

A principal dificuldade manifestada foi a falta de clareza dos gráficos de indicadores [D01]. Essa dificuldade está associada à recomendação de ajustes na forma de apresentação dos indicadores [S01].

"O resultado dos indicadores está um pouco confuso." [D01].

"Talvez melhor distribuição das barras dos indicadores." [S01].

"A cor para os indicadores com desempenho abaixo dos alunos aprovados deveria ser mais chamativa." [S01].

No campo do dashboard que é reservado ao registro de impedimentos do usuário para a realização das atividades propostas na disciplina, foi recomendado acrescentar a opção de arquivos anexos [S02], o que permitiria o envio de comprovantes dos obstáculos enfrentados pelos estudantes.

"O campo existem "impedimentos para suas atividades" poderia ter a opção de adicionar anexos (documentos, imagens, ...), visto que há possibilidade de algum aluno sentir a necessidade de adicionar alguma declaração mais formal." [S02].

\subsection{Análise dos dados coletados por meio do questionário SUS}

Como outra fonte de evidências da percepção dos usuários a respeito da usabilidade do recurso testado, foram coletados dados por intermédio do questionário SUS. Para a análise desses dados, adotaram-se os parâmetros de medida das respostas do SUS definidos por Brooke et al. (1996) e a classificação dos resultados recomendada por Bangor et al. (2009).

O gráfico da Figura 6 aponta os resultados, individuais e médio, da avaliação dos usuários acerca do dashboard. A pontuação média relativa ao artefato foi 89,04 , a qual permite classificá-lo como "bom", sob o ponto de vista da usabilidade. Os resultados 
mostram que, enquanto 38 usuários qualificaram esse artefato como "aceitável", "bom" ou "excelente", apenas 1 usuário considerou o recurso "com problemas de usabilidade".

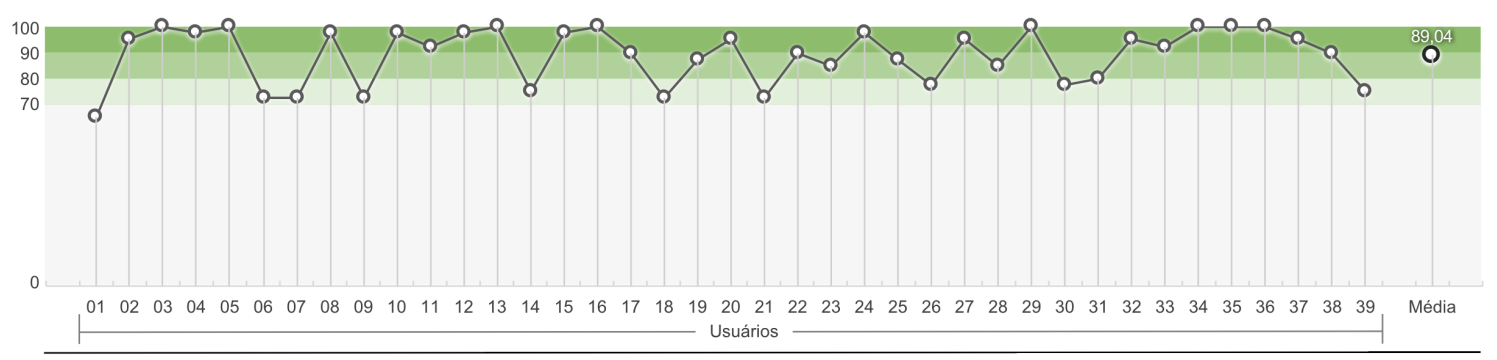

Frequência de resultados por classificação: $\quad 3 \%$ Com problemas de usabilidade $(N=1) \quad 23 \%$ Aceitável $(N=9) \quad 13 \%$ Bom $(N=5) \quad 62 \%$ Excelente $(N=24)$

Figure 6. Resultado do SUS para o dashboard avaliado na pesquisa.

A Figura 7 oferece outra perspectiva para a compreensão dos resultados, com a distribuição de frequência das respostas em cada item do SUS. Todos os participantes concordaram com as declarações D01 - "Eu acho que gostaria de utilizar esse recurso frequentemente" e D03 - "Eu achei o recurso fácil de usar". Por outro lado, 1 dos respondentes concordou com a D04 - "Eu acho que precisaria do apoio de um suporte técnico para usar esse recurso" e outros 4 foram neutros em relação à $\mathbf{D 0 9}$ - "Eu me senti muito confiante usando esse recurso" (Figura 7). Esses resultados estão alinhados com a dificuldade enfrentada por alguns estudantes para a interpretação dos gráficos de indicadores do boletim, a qual foi relatada na Seção 4.2.

\begin{tabular}{|c|c|c|c|c|c|c|c|c|c|c|c|c|c|c|}
\hline \multirow{3}{*}{ Declaraçōes } & \multicolumn{10}{|c|}{ Distribuição de frequência das respostas* } & \multirow{3}{*}{$100 \%$} & \multirow{3}{*}{$\begin{array}{c}\begin{array}{c}\text { Percentual de } \\
\text { respostas negativas } \\
\text { ou neutras }\end{array} \\
\end{array}$} & \multirow{3}{*}{$\begin{array}{l}\text { Percentual de } \\
\text { respostas positivas }\end{array}$} & \multirow{3}{*}{$\stackrel{100 \%}{\longrightarrow}$} \\
\hline & \multicolumn{2}{|c|}{1} & \multicolumn{2}{|c|}{2} & \multicolumn{2}{|c|}{3} & \multicolumn{2}{|c|}{4} & \multicolumn{2}{|c|}{5} & & & & \\
\hline & $\mathrm{N}$ & $\%$ & $\mathrm{~N}$ & $\%$ & $\mathrm{~N}$ & $\%$ & $\mathrm{~N}$ & $\%$ & $\mathrm{~N}$ & $\%$ & & & & \\
\hline $\begin{array}{l}\text { Eu acho que gostaria de utilizar esse recurso } \\
\text { frequentemente [D01] }\end{array}$ & 0 & $0 \%$ & 0 & $0 \%$ & 0 & $0 \%$ & 18 & $46 \%$ & 21 & $54 \%$ & & $0 \%$ & & $100 \%$ \\
\hline $\begin{array}{l}\text { Eu achei o recurso desnecessariamente complexo } \\
\text { [D02] ** }\end{array}$ & 25 & $64 \%$ & 13 & $33 \%$ & 1 & $3 \%$ & 0 & $0 \%$ & 0 & $0 \%$ & & $3 \%$ & & $97 \%$ \\
\hline Eu achei o recurso fácil de usar [D03] & 0 & $0 \%$ & 0 & $0 \%$ & 0 & $0 \%$ & 14 & $36 \%$ & 25 & $64 \%$ & & $0 \%$ & & $100 \%$ \\
\hline $\begin{array}{l}\text { Eu acho que precisaria do apoio de um suporte técnico } \\
\text { para usar esse recurso [D04] ** }\end{array}$ & 24 & $62 \%$ & 14 & $36 \%$ & 0 & $0 \%$ & 1 & $3 \%$ & 0 & $0 \%$ & & $3 \%$ & & $97 \%$ \\
\hline $\begin{array}{l}\text { Eu achei que as funções desse recurso foram bem } \\
\text { integradas [D05] }\end{array}$ & 0 & $0 \%$ & 0 & $0 \%$ & 2 & $5 \%$ & 15 & $38 \%$ & 22 & $56 \%$ & & $5 \%$ & & $95 \%$ \\
\hline $\begin{array}{l}\text { Eu achei que o recurso apresenta muita inconsistência } \\
\text { [D06] ** }\end{array}$ & 26 & $67 \%$ & 12 & $31 \%$ & 1 & $3 \%$ & 0 & $0 \%$ & 0 & $0 \%$ & & $3 \%$ & & $97 \%$ \\
\hline $\begin{array}{l}\text { Eu imagino que a maioria das pessoas aprenderia a } \\
\text { usar esse recurso rapidamente [D07] }\end{array}$ & 0 & $0 \%$ & 0 & $0 \%$ & 2 & $5 \%$ & 18 & $46 \%$ & 19 & $49 \%$ & & $5 \%$ & & $95 \%$ \\
\hline Eu achei o recurso muito estranho de usar [D08] ** & 27 & $69 \%$ & 12 & $31 \%$ & 0 & $0 \%$ & 0 & $0 \%$ & 0 & $0 \%$ & & $0 \%$ & & $100 \%$ \\
\hline $\begin{array}{l}\text { Eu me senti muito confiante usando esse recurso } \\
\text { [D09] }\end{array}$ & 0 & $0 \%$ & 0 & $0 \%$ & 4 & $10 \%$ & 15 & $38 \%$ & 20 & $51 \%$ & & $10 \%$ & & $90 \%$ \\
\hline $\begin{array}{l}\text { Eu precisei aprender uma série de coisas antes que eu } \\
\text { pudesse continuar a utilizar esse recurso [D10] ** }\end{array}$ & 22 & $56 \%$ & 17 & $44 \%$ & 0 & $0 \%$ & 0 & $0 \%$ & 0 & $0 \%$ & & $0 \%$ & & $100 \%$ \\
\hline
\end{tabular}

Figure 7. Frequência de respostas da avaliação.

\section{Considerações Finais}

A metodologia Sala de Aula Invertida (SAI) tem se revelado um modelo mais promissor do que o processo tradicional de ensino. Pesquisas apontam que essa metodologia, combinando atividades a distância e práticas ativas, tem um grande potencial para melhorar o desempenho acadêmico dos estudantes e reduzir a evasão escolar. Porém, a necessidade de um corpo discente qualificado, com a devida capacidade para gerir os seus estudos, é um aspecto que 
compromete a aprendizagem invertida, o qual torna a Autorregulação da Aprendizagem (ARA) um desafio crítico para o sucesso da SAI.

A motivação deste trabalho reside, exatamente, no desafio referenciado acima. Diante de um número crescente de estudos que apresentam as soluções de Learning Analytics (LA) como um mecanismo de promoção da ARA, o objetivo deste estudo foi avaliar a usabilidade de um dashboard destinado à ARA em SAI.

$\mathrm{Na}$ seção 4, foram discutidos os resultados dos testes de usabilidade do recurso. Mesmo com pouco tempo para se acostumarem com todas as funcionalidades, trinta e nove (39) usuários executaram os testes sem grandes dificuldades e mostraram, não apenas reconhecer a qualidade de usabilidade, mas também a importância do recurso para a melhoria do processo de aprendizagem. Os usuários, ainda, fizeram algumas sugestões pertinentes, as quais podem ser utilizadas na evolução do artefato, ampliando o potencial de apoio do recurso de LA na ARA.

A partir das sugestões apresentadas pelos participantes da pesquisa, em trabalhos futuros, pretende-se realizar ajustes no dashboard avaliado neste estudo e utilizá-lo em novos experimentos, para averiguar os impactos do artefato sobre a ARA em SAI.

\section{Referências bibliográficas}

ASSILA, A. et al. Standardized usability questionnaires: Features and quality focus. Electronic Journal of Computer Science and Information Technology, v. 6, n. 1, 2016.

BANGOR, A.; KORTUM, P.; MILLER, J. Determining what individual SUS scores mean: Adding an adjective rating scale. Journal of usability studies, v. 4, n. 3, p. 114-123, 2009.

BROOKE, John et al. SUS-A quick and dirty usability scale. Usability evaluation in industry, v. 189, n. 194, p. 4-7, 1996.

CAPES. (2010). Fomento à inovação para o desenvolvimento e aplicação de Tecnologias de Informação e Comunicação em Educação. Disponível em: <http://www.capes.gov.br /images/stories/download/editais/resultados/RESULTADO_FINAL_EDITAL_15_DED_C APES_25.05.2010.pdf>. Acesso em: 02 outubro 2018.

CAPES. (2015). Fomento à inovação para o desenvolvimento e aplicação de Tecnologias de Informação e Comunicação em Educação. Disponível em: <http://www.capes.gov.br /images/stories/download/editais/resultados/100552016- resultado-032015-UAB-final.pdf>. Acesso em: 02 outubro 2018.

CORRIN, L.; BARBA, P. How do students interpret feedback delivered via dashboards? In: Proceedings of the Fifth International Conference on Learning Analytics \& Knowledge. ACM, 2015. p. 430-431.

DAVIS, D. et al. Encouraging Metacognition \& Self-Regulation in MOOCs through Increased Learner Feedback. In: Proceedings of the sixth International Conference on Learning Analytics \& Knowledge. ACM, 2016. p. 17-22.

DELOZIER, S.; RHODES, M. Flipped classrooms: a review of key ideas and recommendations for practice. Educational Psychology Review, p. 1-11, 2016.

DURALL, Eva; GROS, Begoña. Learning Analytics as a Metacognitive Tool. In: CSEDU (1). 2014. p. 380-384.

FELDER, R. et al. Learning and teaching styles in engineering education. Engineering education, v. 78, n. 7, p. 674-681, 1988. 
FIDALGO-BLANCO, A. et al. Micro flip teaching-An innovative model to promote the active involvement of students. Computers in Human Behavior, v. 72, p. 713-723, 2017. FLICK, U. Introdução à Pesquisa Qualitativa. Artmed Editora, 2009.

FREEMAN, Scott et al. Active learning increases student performance in science, engineering, and mathematics. Proceedings of the National Academy of Sciences, v. 111, n. 23, p. 8410-8415, 2014.

KARAOĞLAN YILMAZ, F.; OLPAK, Y.; YILMAZ, R. The Effect of the Metacognitive Support via Pedagogical Agent on Self-Regulation Skills. Journal of Educational Computing Research, p. 0735633117707696, 2017.

LAGE, M. J.; PLATT, G. J.; TREGLIA, M. Inverting the classroom: A gateway to creating an inclusive learning environment. The Journal of Economic Education, v. 31, n. 1, p. 30-43, 2000.

MASON, G.; SHUMAN, T.; COOK, K. Comparing the effectiveness of an inverted classroom to a traditional classroom in an upper- division engineering course. IEEE Transactions on Education, v. 56, n. 4, p. 430-435, 2013.

MAZUR, Eric. Peer instruction: getting students to think in class. In: AIP Conference Proceedings. AIP, 1997. p. 981-988.

MAZUR, Eric. The Flipped Classroom Will Redefine the Role of Educators, 2013. Disponível em: <http://evolllution.com/revenue-streams/distance_online_learning/audioflipped-classroom-redefine-role-educators-10-years/>. Acesso em: 02 outubro 2018.

O'FLAHERTY, Jacqueline; PHILLIPS, Craig. The use of flipped classrooms in higher education: A scoping review. The Internet and Higher Education, v. 25, p. 85-95, 2015.

PREECE, J.; ROGERS, Y.; SHARP, H. Design de interação. bookman, 2005.

RODRIGUES, R. et al. Uma Abordagem de Regressão Múltipla para validação de variáveis de Autorregulação da aprendizagem em ambientes de LMS. In: Anais do Congresso Brasileiro de Informática na Educação, 2016, Uberlândia.

SIEMENS, George; D BAKER, Ryan SJ. Learning analytics and educational data mining: towards communication and collaboration. In: Proceedings of the 2nd international conference on learning analytics and knowledge. ACM, 2012. p. 252-254.

SUN, Z.; LU, L.; XIE, K. The Effects of Self-Regulated Learning on Students' Performance Trajectory in the Flipped Math Classroom. In: 12th International Conference of the Learning Sciences. International Society of the Learning Sciences, 2016. p. 66-73.

TABUENCA, B. et al. Time will tell: The role of mobile learning analytics in selfregulated learning. Computers \& Education, v. 89, p. 53-74, 2015.

TORI, Romero. Cursos híbridos ou blended learning. In: LITTO, Fredric M.; FORMIGA, Marcos (org.). Educação a Distância: O Estado da Arte. São Paulo: Pearson Education do Brasil, 2009. p. 121-128.

VALENTE, José Armando. Blended learning e as mudanças no ensino superior: a proposta da sala de aula invertida. Educar em Revista, p. 79-97, 2014.

VERBERT, Katrien et al. Learning analytics dashboard applications. American Behavioral Scientist, v. 57, n. 10, p. 1500-1509, 2013.

ZIMMERMAN, B. J. Attaining self-regulation: A social cognitive perspective. Handbook of Self-Regulation. New York: Academic Press, p. 13-39, 2000. 